\title{
Improvement of defect detection in shearography by using Principal Component Analysis
}

\author{
Jean-François Vandenrijt, Nicolas Lièvre, Marc P. Georges*, \\ Centre Spatial de Liège, Université de Liège, Liege Science Park, B-4031 Angleur (Liege), Belgium
}

\begin{abstract}
A post-processing technique based on principal components analysis (PCA) is proposed for shearography for defect detection. PCA allows decomposing a time series of images into a set of images called Empirical Orthogonal Functions (EOF), each showing features with a given variability in the time series. We have applied PCA on composite samples containing various defects at different depths and which undergo transient thermal wave. Analyzing the temporal series shows the shallow defects appearing first whereas the deeper ones appear later. With PCA all the defects appear in one or two of the EOF, easing the identification of defects.
\end{abstract}

Keywords: fringe analysis technique, principal component analysis, shearography, nondestructive testing, defect detection

\section{INTRODUCTION}

Shearography ${ }^{[1]}$ is a non invasive technique which is mainly used for detecting the presence of damages/defects underneath the surface of objects. Shearography records the interference pattern between a speckle object wavefront and itself laterally displaced through an optical shearing device. Such interference patterns, so-called shearograms, are recorded at different instants between which the inspected object undergoes a certain stress (thermal, mechanical, pressure variation, vibration). The numerical difference between two shearograms gives rise to an interferogram which allows observing the derivative of the full-field displacement of the object under the stress. The presence of defect induces a specific local signature in the interferogram which allows localizing the defect under the surface.

One of the most and simplest way of object stimulation for defect detection is heating with lamps. The visibility of defects signatures in interferogram depends on many factors like the material, the nature of the defect, its extent and depth. This renders quite difficult the procedure for finding the best way to stress the object. In the case of a thermal stress, one usually uses the step heating: a few seconds heating and the object is let under thermal relaxation, the interferograms being observed during the thermal relaxation.

When several defects are present at different depths, the shallow ones are visible just at the beginning of the observation sequence, whereas the deeper ones appear later. Therefore it is of high interest to capture a sequence of interferograms and to search for the different defects by analyzing the different images of the sequence. This process can become quite long and difficult, moreover if the

The Principal Components Analysis (PCA) is a statistical processing method of which mathematical concept consists of reducing the dimensionality of a data set which is made of a large number of interrelated variables, while retaining the variation present in the data $\mathrm{set}^{[2]}$. The initial set of variables is transformed into a new set of variables called the principal components, which are uncorrelated and ordered in such a way that the first ones contain the most of the variation present in all of the original variables. The number of principal components is less or equal to the number of original variables, but in practice the method is used to reduce the number of original variables. PCA is applied in a wide variety of domains, in science (e.g. spectroscopy ${ }^{[3]}$ ), or multimedia for face recognition ${ }^{[4]}$. PCA is currently successfully applied to full-field NDT by thermography ${ }^{[5][6][7]}$ and for that reason we wanted to study its application to shearography.

\footnotetext{
* mgeorges@ulg.ac.be; http://www.csl.ulg.ac.be
} 
In this paper we will briefly recall the basics of shearography, and then we explain the PCA methods and how we apply it to shearography temporal series of data. Finally we show an example of application in defect detection in composites.

\section{BASICS OF SHEAROGRAPHY}

The principle of working of shearography is shown in Figure 1(a). A laser illuminates the sample to be inspected. The latter scatters light towards the shearing device which consists of a beamsplitter cube (BC) which splits the object image in two parts which are retroreflected by mirrors M1 and M2 before entering a camera. If mirrors M1 and M2 are perpendicular one another, both images are perfectly superimposed in the image plane. If one of the mirror is slightly tilted by an angle $\alpha$ (mirror M2 in Figure 1), both reflected images appear transversally displaced one another (Figure $1(b))$.

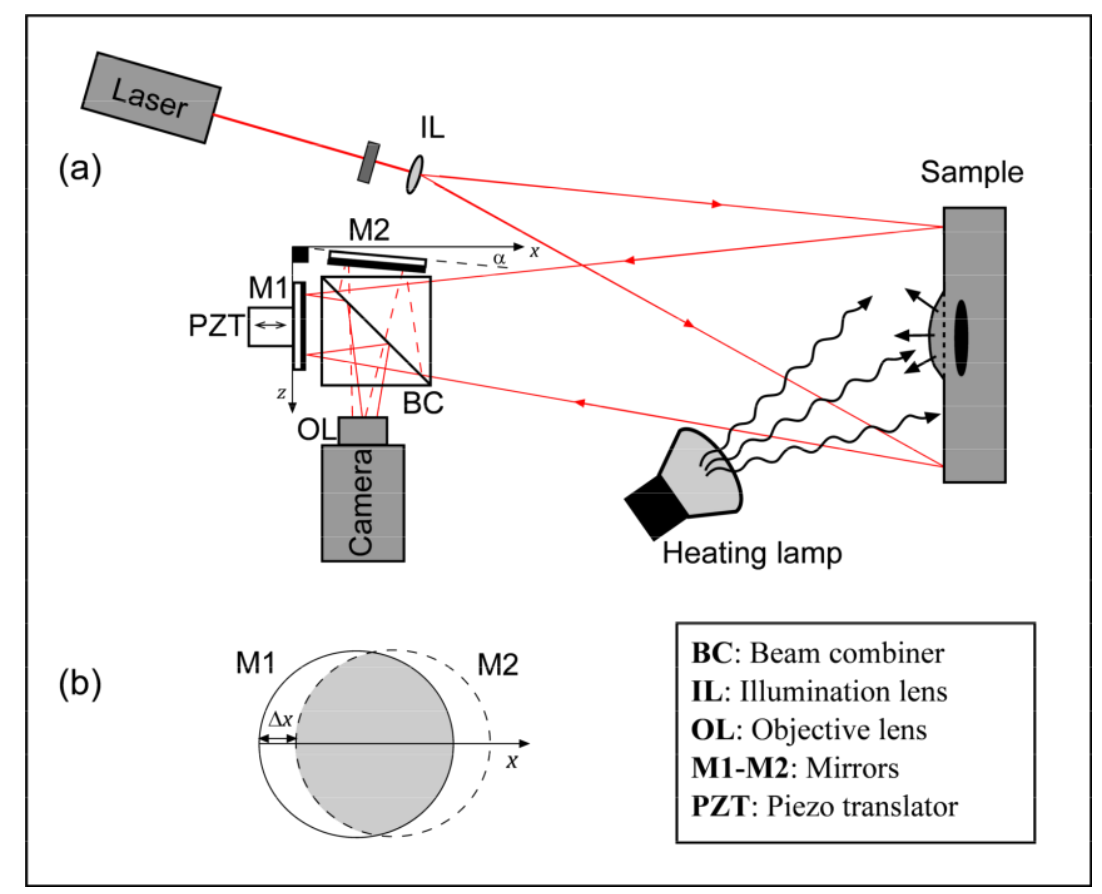

Figure 1. (a) Shearography set-up; (b) Overlap of transversally displaced images

Where both images overlap in the image plane (the grey area in Figure 1(b)), interference occurs and gives rise to a socalled shearogram whose intensity profile in each pixel located in the camera plane can be described as

$$
I=I_{O, 1}+I_{O, 2}+2 \sqrt{I_{O, 1} I_{O, 2}} \cos \phi
$$

where $I_{O, 1}$ and $I_{O, 2}$ are the intensities of object waves reflected respectively by mirror M1 and M2, and which are displaced one another in the $x$ direction (shear direction) by an amount $\Delta x$ (shear amplitude). The phase can be computed if one considers the phase-shifting technique ${ }^{[8]}$ which consists of acquiring a set of shearograms with an additional known phase step between each frame capture. For that purpose a piezoelectric translator (PZT) moves mirror M1 to produce a given phase step. Usually we use the 4 -frame algorithm with a $\pi / 2$ phase step, which gives

$$
I_{i}=I_{O, 1}+I_{O, 2}+2 \sqrt{I_{O, 1} I_{O, 2}} \cos (\phi+(i-1) \pi / 2)
$$

with $\mathrm{i}=1,2,3,4$. The phase $\phi$ can be computed following the following formula:

$$
\phi=\tan ^{-1}\left(\frac{I_{4}-I_{2}}{I_{1}-I_{3}}\right)
$$

The modern application of shearography to NDT requires the acquisition of series of 4 phase-shifted shearograms at different instants. Usually a first set is taken to provide the reference state and a reference phase $\phi_{r}$ is computed by (3). During the object stress, similarly one can determine the phase $\phi$. Then it is sufficient to subtract $\phi_{r}$ to the phase $\phi$ at each instant to obtain a phase variation which can be related to the strain undergone by the object and given by 


$$
\Delta \phi=\frac{2 \pi}{\lambda} s \frac{\partial d}{\partial x} \Delta x
$$

where $s$ is the sensitivity vector, $\partial \boldsymbol{d} / \partial x$ and $\Delta x$ are respectively the strain component and the shear amount in the $x$ direction.

\section{APPLICATION OF PCA TO SHEAROGRAPHY}

In shearography we have to analyze the evolution of the content of phase images which appear at different consecutive instants $t_{k}$. The situation looks like in Figure 2. Such image, consists of $N_{x}$ rows and $N_{y}$ columns of pixels obtained at different instants $t_{k}$, with $k=1$ to $N_{t}, N_{t}$ being the total number of shearography results.

In order to apply PCA, one needs to rearrange the 3D matrix into a 2D one. The pixels ensemble consists of a set of spatial variables which can be rearranged on a single line with length $N_{s}=N_{x} \times N_{y}$, while the value of each pixel is arranged in a column with length $N_{t}$. This gives rise to a new 2D matrix with space in one direction and time in the other.

\section{Time series of experimental images}

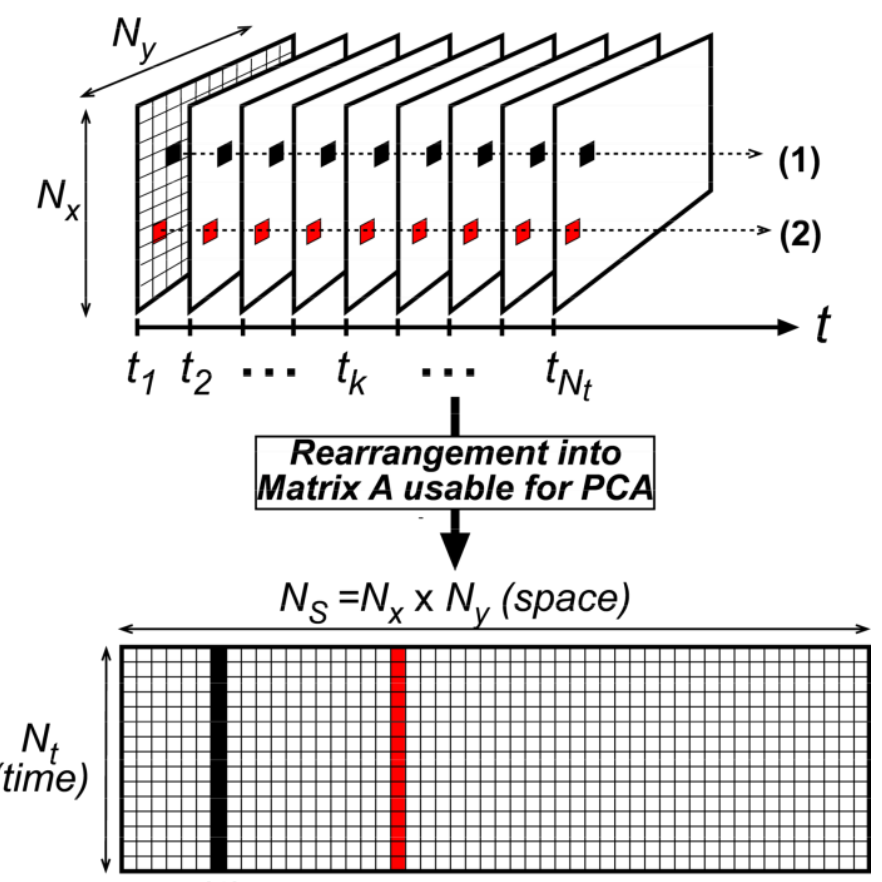

(1)

(2)

Figure 2. Rearrangement of temporal series of images into a 2D matrix usable for PCA

The principal components, or EOFs, are the eigenvectors of the covariance matrix which is the product of the transposed matrix $A$ and $A$ itself, so we can write

$$
\left(A^{T} \cdot A\right) \cdot E O F=a \cdot E O F
$$

where $a$ is the eigenvalue related to a given eigenvector EOF. The calculated eigenvalues are ranked by decreasing values and the corresponding eigenvectors calculated.

In practice there are different ways to compute the EOF. In thermography series analysis by PCA (the so-called Principal Component Thermography - PCT), Rajic ${ }^{17]}$ makes use of singular value decomposition (SVD) of matrix A, which is an alternative to the classical PCA method exposed above. However the results are similar. 
In our case we remarked that the covariance matrix $A^{T} . A$ has a $N_{S} \times N_{S}$ dimension. We developed the PCA under Matlab and it appeared soon that the calculation of eigenvalues and eigenvectors of such a huge matrix was time consuming. We then used a trick to lower the calculation time. It consists in considering the reversed product of matrices $\left(A . A^{T}\right)$ which gives rise to a matrix with dimension $N_{t} \times N_{t}$, which is much smaller than the covariance matrix. This matrix has its own eigenvectors $f^{\prime}$ and eigenvalues $a^{\prime}$ such that

$$
\left(A \cdot A^{T}\right) \cdot f^{\prime}=a^{\prime} \cdot f^{\prime}
$$

and which can be easily computed through Matlab. In order to recover the EOF, we have to add a transformation. If we multiply this by the transposed matrix, it comes

$$
A^{T} \cdot\left(A \cdot A^{T}\right) \cdot f^{\prime}=A^{T} \cdot a^{\prime} \cdot f^{\prime}=a^{\prime} \cdot A^{T} \cdot f^{\prime}
$$

Rewriting the bracket differently, we obtain

$$
\left(A^{T} \cdot A\right) \cdot\left(A^{T} \cdot f^{\prime}\right)=a^{\prime} \cdot\left(A^{T} \cdot f^{\prime}\right)
$$

By similarity between (5) and (8), we can compute the EOF, having found the eigenfunctions $f^{\prime}$ from (6). The EOF are vectors with dimension $N_{s}$. These vectors can be segmented and rearranged to obtain images of dimensions $N_{x} \times N_{y}$.

In order to apply PCA to a temporal series of phase images obtained, we need to pay attention to the following. When the lamp heating method is used, the temperature changes monotonically with time. Applying PCA to the case of thermography images is then quite straightforward and gives useful results. This is not the case in interferometric type of measurements because the intensity of pattern is sinusoidally varying, or if phase is computed, it experiences $2 \pi$ phase jumps. Therefore the PCA cannot be applied directly on a set of interferometric data.

Before applying PCA we need to avoid any sudden phase change between the different images, which are phase images. In our set-up we perform the phase-shifting (e.g. the 4-step algorithm) which allows obtaining phase map between different instants. Additionally we need to relate successive phase images by applying also the temporal phase unwrapping. Temporal unwrapping ${ }^{[9]}$ allows one to follow displacement of each pixel in time as long as the phase variation between each acquisition is smaller than $\pi$.

Once the temporal sequence of phase images is prepared, it is rearranged in a 2D matrix as explained earlier.

\section{RESULTS}

In order to illustrate the use of PCA, we have carried out a typical experiment of defect detection with shearography in a composite sample. The latter is a sandwich panel with foam core and a variety of inclusions of debondings between the skins and the core. A heating lamp is placed on the back side of the sample. A reference shearogram is recorded and phase-shifting is applied to compute the reference phase state. Then the heating occurs for a few seconds (step heating) and a series of phase-shifted acquisitions is performed over some time (typically 30 seconds to 1 minute). Phase interferograms can then be computed by simply subtracting the phase at different subsequent instants to the reference phase. Figure 3 shows 4 phase interferograms out of a sequence of few hundreds. The phase is displayed here modulo $2 \pi$ (shown without spatial phase unwrapping). The first interferogram on the left shows a global behavior in the derivative of displacement field at the beginning of heating. The next interferogram shows the deformation induced by the heat wave and shows both a global behavior (the 4 horizontal fringes) and some local variations to the fringes, which come from the presence of inserts inside the core). In the third image the global deformation has decreased significantly, the inclusions appear better while new local defects appear on top and bottom of the image. In the last image, the global deformation has disappeared and two important defects appear on the top and bottom.

This example shows that during the travel of a heat wave through a sample, the interferograms vary quite significantly with time and defects located at different depths are visible at some specific instants. Here the deeper ones appear first because they are hit by the thermal wave first. Therefore they provoke a local deformation at these instants. The shallow defects appear at the end of the sequence, while the previous ones have disappeared.

This shows also how complex can be the analysis of such a sequence when many defects are present at different depths. 

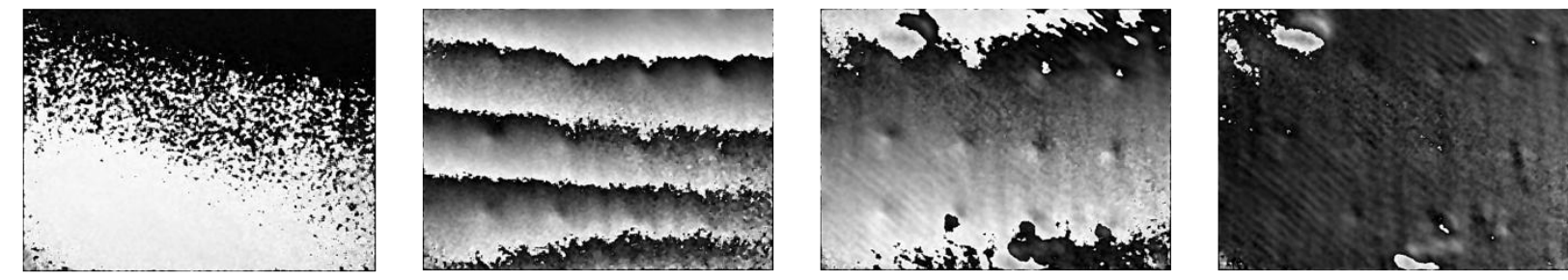

time

Figure 3. Shearographic interferograms obtained at different instants during the passage of a heat wave through a composite sandwich panel with defects at different depths.

We have applied the PCA to the complete sequence and obtained various EOFs. The first and second order EOFs are the only one of interest and are shown hereafter.

(a)

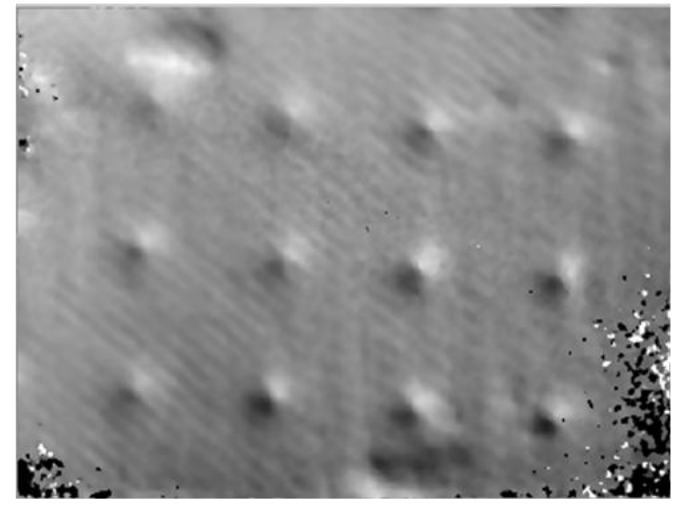

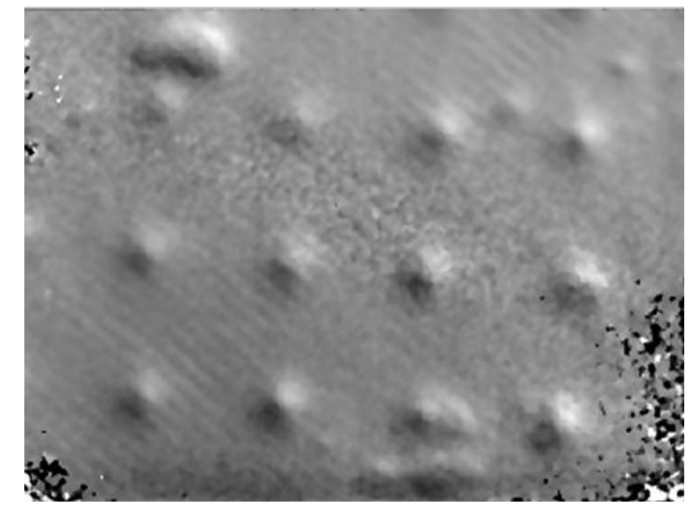

(b)

Figure 4. (a) first EOF, (b) second EOF of the temporal series

We easily see that the different defects which appear at different instants of the temporal series appear as equivalently variable events in the spatio-temporal data sets and are ranged together in the 2 first EOFs.

We find this quite interesting in practice if one wishes to automate the search for defects which can become much easier if the useful data are represented by a few images, instead of by a huge data set.

\section{CONCLUSION}

For the first time, to the best of our knowledge, we have applied the Principal Component Analysis (PCA) to the shearography technique. PCA is a statistical method which allows finding the variability in a series of experiments or data set. Here the data set is constituted by a temporal series of phase images obtained during the thermal decay of a composite sandwich panel containing internal defects and which undergoes a step heating. Various defects are present at different depths and their interferometric signatures appear at different instants.

We have shown that PCA applied to shearography allows retrieving Empirical Orthogonal Functions (EOFs) which are eigenvectors of a covariance matrix built on the basis of the temporal series of phase images. The first order EOF shows in a single image all the defects which otherwise appear at different instants during the time series.

Therefore PCA appears as a useful tool for helping detection of defects. In the future we will experiment PCA on a wider variety of composite samples, with various types of defects. 


\section{REFERENCES}

[1] Steinchen, W., Yang, L., [Digital Shearography], SPIE (2003)

[2] Joliffe, I.T., [Principal Components Analysis, Second Edition], Springer (2002).

[3] Rasmussen, G.T., Isenhour, T.L., "Principal component analysis of the infrared spectra of mixtures," Analytica Chimica Acta 103(3), 213-221 (1978)

[4] Yang, J., Zhang, D., Frangi, A.F., "Two-dimensional PCA: a new approach to appearance-based face representation and recognition," IEEE Trans. On Pattern Analysis and Machine Intelligence, 26(1), 131-137 (2004)

[5] Maldague, X., [Theory and Practice of Infrared Technology for Nondestructive Testing], Wiley Interscience (2001)

[6] Ibarra-Castanedo, C., Avdelidis, N.P., M. Grenier., Maldague, X., Bendada, A., "Active thermography signal processing techniques for defect detection and characterization on composite materials," Proc. SPIE 7661, 766100 (2010)

[7] Rajic, N., "Principal component thermography for flaw contrast enhancement and flaw depth characterization in composite structures," Composite Structures 58, 521-528 (2002)

[8] Creath, K., "Phase-measurement interferometry techniques," Prog. Opt. XXVI, 349-398 (1988)

[9] Huntley, J.M., Saldner, H., "Temporal phase-unwrapping algorithm for automated interferogram analysis," Appl. Opt. 32(17), 3047-3052 (1993) 